\title{
EDUCACIÓN MAPUCHE E INTERCULTURALIDAD: UN ANÁLISIS CRÍTICO DESDE UNA ETNOGRAFÍA ESCOLAR
}

\author{
MAPUCHE EDUCATION AND INTERCULTURALITY: \\ A CRITICAL ANALYSIS FROM A SCHOOL-BASED ETHNOGRAPHY
}

\author{
Laura Luna Figueroa ${ }^{1}$
}

\begin{abstract}
Este artículo se propone contribuir, desde la antropología de la educación y la etnografía escolar, al debate sobre la formación de niños mapuches e indígenas en contexto escolar a partir del caso de una escuela rural en la región de la Araucanía, que se define en el discurso de sus profesores como "no intercultural". Esta escuela particular subvencionada se ha apropiado del espacio escolar para un proyecto de "educación mapuche" vinculado a un proyecto político de autodeterminación. En este trabajo se argumenta que, pese a los discursos anti-interculturales y esencialistas, la escuela se configura como una comunidad de práctica donde conviven elementos y mundos de significados diferentes en una lógica bicultural. Esta convivencia bicultural junto con la predominancia del modelo escolar tradicional, representan un bagaje formativo significativo para los niños que hace de contrapeso al trabajo de concientización sobre el ser mapuche. Se reflexiona sobre la funcionalidad de una visión esencialista de la cultura mapuche para el empoderamiento social y político y se sugiere que el esencialismo cultural podría ser más perjudicial que beneficioso para el logro de este valioso proyecto formativo.
\end{abstract}

Palabras claves: interculturalidad, educación mapuche, comunidad de práctica, etnografía escolar.

This article, based on the study of a rural school, is intended as a contribution, from educational anthropology and school ethnography, to the debate on the school education of Mapuche and other indigenous children. The rural school studied is a charter school located in the Araucanía Region, and is defined by its teachers as "non-intercultural". This educational center has appropriated the school space in order to develop a project of "Mapuche education" linked to a political agenda of selfdetermination. In this paper, it is argued that, despite the anti-intercultural and essentialist discourses, the school is a community of practice where different elements and meanings coexist in a bicultural logic. This bicultural coexistence and the predominance of the traditional school model represent for children a significant knowledge background that counterbalances teachers' work on raising consciousness about being Mapuche. The functionality of an essentialist vision of Mapuche culture aimed at social and political empowerment is discussed, suggesting that cultural essentialism might be more detrimental than beneficial for the success of this valuable educational project.

Key words: Interculturality, Mapuche education, school ethnography, community of practice.

La Antropología de la Educación en Chile se encuentra aún en un estado muy incipiente. Subdisciplina de la antropología cultural y social, esta surge de la escuela norteamericana de Cultura y Personalidad de los años Treinta interesada en investigar sobre la influencia de "patrones culturales" en las formas de comportamiento individual (Benedict 1934). En este marco fue pionera la obra de Margaret Mead (1928) porque puso atención específicamente en los procesos de socialización que llevan al individuo a adquirir su cultura. Posteriormente, en los años cincuenta y, especialmente bajo la influencia de Luis Spindler (1955), la antropología de la educación se establece como un campo de estudios dedicado al análisis antropológico de los procesos de transmisión cultural no solo en contextos no formales, sino también dentro de instancias formales de formación, y particularmente, en el marco de la educación escolar.

En Latinoamérica, empezando por México, el involucramiento de los antropólogos en educación cuenta con varias décadas de historia, al estar vinculado a su interés en los procesos de asimilación cultural de los indígenas y a su apología de los derechos culturales y lingüísticos de los pueblos originarios (Rockwell y Gomes 2009). Sin embargo, la realización de estudios antropológicos sobre educación indígena es reciente (Rockwell 2011); más aún, la aproximación antropológica, por medio de la etnografía, a los procesos sociales que ocurren con el fenómeno de la escolarización no se afirma antes de los años ochenta, especialmente gracias al

$1 \quad$ Pontificia Universidad Católica de Chile, Campus Villarrica. O’Higgins 501, Villarrica, Chile. 1luna@uc.cl

Recibido: abril 2014. Aceptado: octubre 2014.

http://dx.doi.org/10.4067/S0717-73562015005000040. Publicado en línea: 14-septiembre-2015. 
trabajo de Elsie Rockwell que, desde México, ha marcado el desarrollo de la antropología y educación en Brasil y Argentina (Tosta 2013).

El interés por las formas en que el poder y la agenda nacionalizante del Estado se impone en las comunidades locales, por medio de la institución escolar es un aspecto recurrente en las etnografías educacionales latinoamericanas (Rockwell 2011). Se ponen a luz las estrategias de "resiliencia" o resistencia, explícitas o implícitas, que aparecen en el día a día de las comunidades escolares, al igual que las modalidades de apropiación de "capital simbólico" para fines ajenos a la agenda estatal y, por último, la fuerte presencia de la comunidad local y de sus formas de vida en una organización que parece imponer su formato universal y arrasar con los particularismos (Tallé 2011).

En Chile, pese a la amplia literatura existente sobre educación intercultural bilingüe, y los saberes educacionales mapuches, en algunos casos basada en estudios cualitativos (Quidel 2011; Fuenzalida y Casas 2010; Peña-Cortés et al. 2010; Quilaqueo y Quintriqueo 2010; Rother 2005; Quintriqueo y Torres 2012; Quilaqueo et al. 2014) hay una notoria escasez de trabajos antropológicos y de etnografías sobre la educación escolar en contexto indígena. Los trabajos de Durán (2007), Álvarez-Santullano et al. (2007), Forno et al. (2009), Álvarez-Santullano (2011) sobre la implementación de la Educación Intercultural Bilingüe (EIB) en contexto mapuche son interesantes excepciones.

En particular, los trabajos de Forno et al. (2009) y Álvarez-Santullano (2011) apuntan al carácter hegemónico de la EIB como política de Estado y a la decontextualización de los contenidos que imparte respecto de la realidad que viven las comunidades mapuche-huilliche. Forno et al. (2009) destaca cómo el discurso técnico del Ministerio de Educación respecto de la EIB choca con el enfoque político de las comunidades mapuche, para las cuales la educación es una herramienta necesaria para el desarrollo y la autonomía.

La oposición entre una visión pedagógica de la EIB, propugnada por las políticas estatales, y una de tipo epistemológico y político, defendida por las organizaciones indígenas, es identificada por López (2011) como una de las principales tensiones que explican la actual disconformidad de los movimientos indígenas hacia los diferentes programas de EIB en Latinoamérica. El caso de la escuela que se analiza en este trabajo se enmarca en esta tensión (López
2011), al tratarse de un proyecto educativo que toma distancia de las actuales políticas de EIB en Chile y al buscar, por medio de la educación, la realización de un proyecto político como pueblo.

\section{Enfoque y Metodología de la Investigación}

Este artículo se basa en una etnografía escolar llevada a cabo durante 2012 en una escuela ubicada en una comunidad mapuche en la región de la Araucanía ${ }^{1}$. El enfoque etnográfico implicó la presencia regular en el colegio de la investigadora y de su asistente de uno a dos días semanales entre marzo y octubre. La observación participante en diferentes instancias (clases, recreos, reuniones, actividades extracurriculares, etc.) y la focalización de la observación de clase en un curso (séptimo año de Educación Básica) junto con la realización de extensas entrevistas en profundidad a los profesores, entrevistas semiestructuradas a los niños ${ }^{2}$ y focus groups con los apoderados, permitió abordar de una manera holística la escuela como "comunidad de práctica" (Wenger 1998). Con esta expresión se entiende un grupo organizado en torno a una agenda y actividades comunes cuyo intercambio regular genera un sistema de significados y un modus operandi compartidos. En este marco se ha buscado estudiar el aprendizaje de los niños de una manera más integral respecto a lo que ocurre dentro de los procesos intencionados de enseñanza-aprendizaje. En línea con Lave y Wenger (1991) el aprendizaje no solamente ocurre de manera situada dentro de la práctica social, sino debe considerarse en sí mismo como un aspecto de la práctica y precisamente como una forma de participación y a la vez de negociación de significados que ocurre en las interacciones sociales cotidianas. Desde esta aproximación, en este artículo se muestran en acción las principales estrategias del proyecto formativo de la escuela y el pensamiento que las sustentan y se reflexiona sobre su impacto en el aprendizaje e identidad de los niños.

\section{La Escuela Comunitaria Mapuche We Newen ${ }^{3}$}

La zona donde se encuentra la escuela We Newen se caracteriza por una alta densidad de población mapuche y rural, una identidad étnica marcada junto con la conservación de rasgos culturales y de la lengua y altos niveles de pobreza. Por lo 
mismo, se convirtió, a fines de la década de 1990, en el foco de una serie de programas estatales y no estatales y, a la vez, en el fulcro de movimientos de demanda política. La insatisfacción de parte de un grupo de profesores mapuches con la educación estatal, aun en sus versión "intercultural bilingüe", y la inquietud por transmitir en la enseñanza formal el conocimiento mapuche todavía presente en las comunidades del sector, llevaron al surgimiento de la escuela We Newen.

La escuela We Newen, perteneciendo en un principio al Magisterio de la Araucanía, fue tomada en gestión el año 2005 por la comunidad Toqui Kura y por algunos profesores mapuches con el doble objetivo de mejorar la calidad de la educación que se impartía y de implementar un modelo educacional más acorde con la identidad y el patrimonio cultural de la zona. El cambio de gestión llevó, en pocos años, a un aumento exponencial del número de las matrículas y a un notable mejoramiento de los resultados del Simce (Sistema de Medición de la Calidad de la Educación) con un proyecto educativo centrado en el fortalecimiento de la identidad cultural del niño. Este destaca dentro de las escasas experiencias educativas de gestión indígena en Chile, de las cuales, por lo demás, no se han encontrado antecedentes en la literatura.

Desde la mañana temprano, antes que la campana marque el inicio de las clases, los profesores se encargan de recordarle a cada niño, individualmente, que es mapuche, saludándolo de mano en lengua mapuzungun: "mari mari lamngen"(a las mujeres); "mari mari peñi" (a los hombres). Los niños contestan a tono dirigiéndose a sus profesores no como "tíos" y "tías", como suele ocurrir en las escuelas chilenas, sino como peñi y lamngen.

En la sala de clases los niños están dispuestos con sus respectivas mesas y sillas en semicírculo mirando hacia la pizarra, delante de la cual está la mesa del profesor, dirigida hacia los estudiantes. El lamngen José da inicio a las clases de Lenguaje y Comunicación. Después de pedirle en mapuzungun a un niño que vaya a buscar astillas y leña para hacer fuego en la sala, el profesor realiza el usual rito del saludo en mapuzungun con los estudiantes que responden a tono.

Pasando a la lengua castellana, el profesor habla del kamarikun que ocurrió en el territorio el fin de semana. Explica en qué consiste la ceremonia y, dibujando en la pizarra un círculo, se refiere a la cosmovisión mapuche. Entonces también hace mención de quienes asistieron a la ceremonia, felicitando a los participantes y sus familias. Agrega que otros, al contrario, están alejados de su espiritualidad mapuche debido a que participan en otras religiones que no ven con buenos ojos este tipo de ceremonias. Luego, en voz alta y en frente de todos, se dirige directamente a una estudiante cuya familia es evangélica. Mirándola dice: "como mi lamngen, que camina para otra iglesia". La niña lo mira de vuelta, tiene una pequeña sonrisa como de resignación. Posteriormente, comienza la clase anotando en la pizarra la fecha en mapuzungun $y$, en castellano, el objetivo de la clase acerca de "Los Factores de la Comunicación". Durante la actividad en los cuadernos, el profesor circula por los puestos revisando el trabajo de los niños y apoyándolos individualmente. A veces intercala frases en mapuzungun que los niños responden en castellano.

Para la clase de mapuzungun, los niños se trasladan a la ruka. Contrariamente a los métodos estandarizados con que se abordan todos los contenidos del currículum oficial, los saberes mapuches, desde la lengua al trabajo con la greda y los significados asociados a ella, se abordan con una metodología que se centra en el hacer, donde se plantean al aprendiz situaciones y desafíos reales. Los profesores tienen conciencia de ello y comentan que desde la "pedagogía mapuche" se "aprende haciendo".

A la hora del recreo, los niños se reparten, en su gran mayoría, entre el juego de la pelota en la cancha, los columpios y otros juegos en el patio y los simples paseos de grupos de mujeres escuchando a Chayanne y reguetón por los celulares. Algunas estudiantes de séptimo también escuchan música, pero de un reproductor MP3 conectado al laptop que una de ellas saca de su mochila dentro de la sala.

El día escolar termina a las 16.10 horas para los niños, pero sigue para los profesores que casi todos los días tienen una reunión después del horario de clases, sea para hablar de temas técnico-pedagógicos, para discutir asuntos con los representantes de la comunidad en el Consejo de Educación o para trabajar sobre nuevos proyectos de la escuela como la elaboración de planes y programas propios. En muchas de estas reuniones es posible escuchar una frase que se repite casi como un eslogan: "Nosotros no creemos en la interculturalidad". 


\section{El Proyecto Educativo y la Postura "Anti-intercultural" de la Escuela We Newen}

Las prácticas cotidianas de la escuela We Newen no dan cuenta del significado de las afirmaciones contra la educación intercultural o la interculturalidad. La escuela parece intercultural en varios sentidos. En primer lugar, en el sentido en que normalmente se entiende y se implementa la educación intercultural en Chile y en Latinoamérica en general, como una educación que presta atención a la cultura y lengua de los niños indígenas, dejando un espacio, dentro de la vida escolar y del currículum oficial, para la transmisión de aspectos culturales y la práctica de la lengua. La lógica subyacente es que los niños puedan formarse en ambas culturas y practicar o aprender, tanto el idioma nacional como el indígena. La escuela We Newen responde a este reto con la particularidad de poner, comparativamente con otras escuelas chilenas con similares características, especial énfasis en los contenidos mapuches. Estos no son solamente relegados a las clases del Sector Lengua Indígena, sino que se trabajan también en los espacios curriculares de libre disposición para cada establecimiento y, parcialmente, de manera transversal, es decir, por medio de vinculaciones a contenidos o la práctica, aún ocasional y acotada, del mapuzungun en las asignaturas tradicionales. Esta escuela se inserta entonces en el modelo tradicional de Educación Intercultural Bilingüe que, aún con modalidades y énfasis diferentes, está orientado a la "biculturizacion del curriculum" (Dietz 2012).

Por otra parte, la We Newen es una escuela intercultural también en el sentido más antropológico del término que se refiere a la dimensión de las relaciones entre culturas diferentes. Es lo que Ansión (2007) define como "interculturalidad de hecho" que se caracteriza por la convivencia de personas, símbolos y artefactos pertenecientes a paradigmas culturales distintos. Esta convivencia es evidente en la escuela donde, por ejemplo, coexisten prácticas de "consumo cultural" asociadas a la cultura mapuche como a la cultura nacional o global: las estudiantes de octavo año básico vendiendo completos para recabar fondos para su paseo de curso, vestidas con sus mantas y prendas tradicionales y escuchando música reguetón entregan una imagen de esta hibridación a nivel de la utilización de productos culturales (Canclini 2006). Se agrega a esto que en la escuela circulan, en calidad de visitas o colaboradores, personas no mapuches chilenas y extranjeras, algunos con una presencia relativamente estable como la de la investigadora responsable de esta investigación y su ayudante.

Las entrevistas en profundidad realizadas a los profesores de la escuela esclarecen acerca del significado y fundamento de su postura contraria a la interculturalidad, al dar cuenta de una visión de interculturalidad no como "realidad fáctica", sino como proyecto (Ansión 2007:43). Este proyecto está lejos de ser entendido como de convivencia armónica sobre la base de una relación igualitaria entre diferentes pueblos y culturas, como plantea Ansión (2007). Más bien, el proyecto es de asimilación por parte de un Estado que, utilizando el paraguas discursivo de la interculturalidad, pretende continuar su misión homogeneizadora y nacionalizante hacia el pueblo mapuche. Hoy, esta misión, bajo la apuesta de las políticas de EIB, toma la forma de legitimación de la acotada inserción de contenidos culturales mapuche, teniendo un espacio marginal y un impacto poco significativo dentro del marco curricular y pedagógico establecido por las políticas educacionales. De tal forma, tomar distancia de la interculturalidad, significa contestar las actuales hegemonías culturales que se perpetúan por medio de las políticas educativas y anteponer a la educación intercultural o educación occidental la educación mapuche.

En esta perspectiva, uno de los objetivos centrales del proyecto formativo de la We Newen es lo que, en diversas entrevistas, aparece con los conceptos de "autovaloración" o "valoración cultural" explicados como "asumirse como mapuche" o "autoestima como mapuche". El director de la escuela explica estos conceptos contraponiéndolos a la actitud "culturalista" que a menudo asume la gente de las comunidades mapuche y que ejemplifica como: "hacer nguillatún por hacer no más". En otras palabras, las prácticas culturales, incluso las más significativas como el nguillatún, tienen un valor muy limitado si no se enmarcan en un proyecto colectivo; es decir, si no tienen proyecciones en términos políticos. Esto explica por qué el proyecto educativo mapuche de la We Newen no se reduce a la enseñanza de la lengua y de contenidos culturales, como lo que ocurre desde la EIB, sino se focaliza en la formación de la conciencia étnica en pos de un proyecto que apunta al empoderamiento político-cultural.

El trabajo de concientización de los niños sobre su identidad étnica se configura como una 
estrategia educativa crucial en la We Newen que se despliega mediante la práctica discursiva de los profesores en el transcurso de la vida escolar de los niños. Es decir, que junto con la enseñanza de contenidos culturales, la práctica de la lengua y la generación de instancias para la vivencia directa de componentes culturales por parte de los niños (por ejemplo, el Wetripantu), hay una apuesta importante por la inducción a la reflexividad o racionalización de la propia identidad, especialmente dirigida a los niños de mayor edad-séptimo y octavo año básico. Mediante discursos llevados por algunos profesores en las clases de mapuzungun principalmente, se invita a los niños a tomar conciencia acerca de: (a) la pertenencia al pueblo mapuche; (b) su unicidad y el valor positivo de la forma de ser mapuche; (c) la contraposición entre una forma de ser mapuche asociada al bienestar y una forma no mapuche asociada al malestar psíquico, corporal, espiritual; (d) la histórica relación de dominación de la segunda forma de ser sobre la primera por obra del Estado y de las iglesias, y las consecuencias en términos de deprivación cultural e identitaria para el pueblo mapuche además que económica, social, y política; (e) la necesidad de reapropiación de los elementos que componen la "esencia" del ser mapuche, empezando por el mapuzungun.

Las historias de vida y "recorridos identitarios" de algunos profesores podrían explicar esta preocupación por la toma de conciencia de la propia pertenencia cultural y étnica. Habiendo sufrido el desarraigo por haberse alejado de su comunidad de origen para cursar estudios en instituciones educativas católicas con internado, tomaron distancia de su cultura y dejaron de hablar su idioma durante un tiempo, para reencontrarse con su identidad solo en la vida universitaria. Su propia experiencia, al igual que la de muchos mapuches, los condujo a creer que para la conformación de la identidad étnica, no es suficiente con haberse criado y haber crecido en un ambiente familiar apegado a la práctica de la lengua y de la cultura, porque el impacto con el "mundo" es demasiado poderoso y puede anular todo ese bagaje si no está acompañado por la convicción de ser un ser distinto y de pertenecer a un pueblo no asimilable al Estado chileno. La "apuesta" de la We Newen es que, con esa convicción, los niños formados en esta escuela no van a sucumbir cuando salgan del contexto comunitario y rural y cambien abruptamente de realidad. Deberán adecuarse al medio, pero por lo menos íntimamente no traicionarán lo propio y mantendrán la fuerza interna como para enfrentar de manera transitoria el paso por el mundo (occidental, urbano, tecnológico, etc.) y volver a la dimensión que, "en esencia", les pertenece como mapuche: en el campo, a contacto con la naturaleza y los seres espirituales, con un trabajo regido por los ritmos y tiempos naturales, respetuosos del kimún mapuche y de la sabiduría de los antiguos.

Ahora, la trayectoria de los mismos profesores muestra que ese ideal es solo parcialmente alcanzable. Si bien los profesores vuelven al campo, a trabajar en pos de su cultura y su desarrollo como pueblo, siguen desenvolviéndose entre dos mundos y rigiéndose por los tiempos del Ministerio junto con los tiempos de la naturaleza. Se relacionan con chilenos no mapuches y con extranjeros, con instituciones gubernamentales y privadas, al igual que con universidades. Ciertamente, toda interacción con estas entidades tiene como finalidad el desarrollo y cumplimiento de su proyecto político; pero, independientemente de la finalidad, la práctica cotidiana de interactuar con "el otro mundo", en sus diversas expresiones legitima y normaliza esa relación a los ojos de los niños.

Desde la perspectiva de Lave y Wenger (1991), la escuela We Newen es una "comunidad de práctica" donde el aprendizaje ocurre en el involucramiento de los niños en actividades y situaciones relacionales más que en la recepción de contenidos. Es decir que, junto con reconocer el valor de las prácticas discursivas de concientización cultural, es necesario tener presentes los efectos en la formación de los niños tanto de su experiencia diaria en la escuela, como de las historias de vidas y características que observan en sus profesores. Estos podrían considerarse significativos ejemplos de compromiso por la cultura, la lengua y la causa mapuche desde una dimensión intercultural.

Por otra parte, la utilización de la institución escuela, con todo su bagaje de prácticas, lenguajes, objetos y la circulación de artefactos y símbolos (música, tecnología, etc.) de la cultura nacional, latinoamericana e incluso global, constituye un hecho, una práctica social con una notable carga valórica en la formación de los niños. Si compartimos con Lave y Wenger (1991) que aprender, participar y convertirse en un cierto tipo de personas son aspectos del mismo fenómeno; los niños aprenderían a ser "personas mapuche-interculturales" en esa práctica social: se desplazan entre los dos mundos, dialogan con ellos y pueden apreciar ambos. Entonces, vale 
la pena preguntarse: ¿cuál es el sentido y el alcance de la postura anti-intercultural de los profesores? ¿Cuáles son sus posibles consecuencias?

\section{Una Visión Esencialista de la Cultura Mapuche}

Es tan contradictorio, cierto, vivir en dos mundos (...). Nosotros ya somos interculturales, porque interactuamos entre dos culturas, pero eso es muy ambiguo, es para confundir. Yo creo que aquí uno tiene que pertenecer a una sola cultura y en ese contexto yo creo que tendría que enmarcarse la educación, la formación de los niños. Es mi ideal de educación -es un sueño, no sé si será utopía- formar al niño en su cultura al máximo. Eso quiere decir que pudiera hablar el mapuzungun perfectamente, y eso no quita que pudiera hablar el castellano también, pero sí el mapuzungun. Volver a conocer todos los contenidos mapuches y asumirlos como tales. Trabajar en un espacio y tiempo que considera el mapuche, no hacer un horario rígido ¿no cierto? Porque la vida nuestra no es eso, la vida nuestra no es competitiva, no es esclavizante, nuestra vida es la libertad y cuando nos colocamos en horario no vivimos una libertad, estamos ahí acondicionados: "si no lo hago, si no lo cumplo no estoy actuando bien". Yo creo que eso pertenece a otro mundo, el mundo mapuche no es eso, que te pongan horario de levantada, horario de entrada, horario de... sino que regirnos por cómo lo hacía nuestra gente... la medición del tiempo con conceptos nuestros, liwen, rayantu, panrayantu o sea reconstituir todo eso y vivirlo de esa forma (Raúl, director de la escuela).

De este extracto de entrevista se desprende cómo la interculturalidad, además de ser vista como una fachada de la política asimilacionista del Estado, es también considerada como una dimensión existencial marcada por la vivencia de dos mundos por parte de los sujetos mapuches. Se estima que esta es dañina para la persona mapuche, fuente de confusión y de tensión psicológica y espiritual ${ }^{4}$. Este malestar está relacionado con la traición de la "esencia" del ser mapuche. Es decir, el pensamiento de los profesores está marcado por lo que se concibe en antropología como "esencialismo cultural, una postura cuya afirmación está vinculada al multiculturalismo en cuanto movimiento de defensa de la diversidad cultural y, en particular, a su demanda por "acciones afirmativas" (Dietz 2012).

La objetivación de ciertos elementos culturales y su instrumentalización como "marcadores étnicos" caracteriza los procesos intraculturales de etnogénesis (Dietz 2012:125), donde, con el objetivo de consolidar una identidad común, se fijan aspectos de la cultura propia como nucleares o esenciales para la definición y el reconocimiento de la misma. El esencialismo cultural está basado en la idea de que los comportamientos humanos están determinados culturalmente y que las subjetividades están subsumidas a las culturas a las cuales pertenecen de manera "innata" (Verkuyten 2003). Como muestra el pensamiento expresado desde la Escuela We Newen, el hecho de que no siempre los sujetos actúen en función de esos rasgos culturales se debe, desde esta perspectiva, a una contaminación de la esencia por indeseables influencias externas, lo que entonces llamaría a la necesidad de redescubrirla bajo esas influencias y a purificarla de ellas, para que las personas reencuentren el orden natural de su existencia. Este es el fundamento de la orientación intracultural de algunos grupos étnicos: junto con la defensa de sus derechos hacia el Estado y la sociedad nacional, asumen la misión de "reconvertir" los miembros de su cultura, devolverlos a su esencia; solo al reconectarse con su esencia cultural, los miembros pertenecientes a grupos étnicos o pueblos originarios, pueden llevar adelante una vida plena.

Creer en la esencia cultural significa no solo creer en la existencia de elementos o rasgos objetivos e inmutables en las culturas, sino también concebir las culturas como inconmensurables; es decir, considerar que las diferencias entre ellas son radicales. De allí la relación que algunos antropólogos han establecido entre "esencialismo cultural" y "racismo cultural": las diferencias culturales son asumidas como algo absoluto que justifica el rechazo a la convivencia o interrelación (Grillo 2003).

La crítica al esencialismo cultural difundido en el mundo contemporáneo se ha configurado como una tarea específicamente antropológica (Hannerz 1996, citado en Dietz 2012). Los problemas identificados por los antropólogos en el esencialismo cultural 
no son solo de carácter conceptual, en el sentido que se está advocando por una visión estática de la cultura y por un acoplamiento entre cultura e identidad, sino también de carácter social y político. Tal como plantea Verkuyten (2003), no siempre el esencialismo cultural puede ser beneficioso para los grupos oprimidos, porque la exacerbación de las diferencias entre grupos puede ser utilizada también para la defensa de los órdenes sociales y hegemonías existentes. También se señala el carácter excluyente que puede tener el "esencialismo estratégico" (Spivak 1987), entendido como la demarcación, de parte de un grupo que se percibe como subalterno, de rasgos culturales e identitarios con fines contrahegemónicos. Este deja fuera de las reivindicaciones políticas subjetividades que no pueden reflejarse en las definiciones impuestas de la cultura en cuestión (Mattío 2009). Con el esencialismo se genera una autoimagen prístina de la propia cultura que invisibiliza las inconsistencias y los puntos críticos (Hviding 1993). Esta visión provoca una postura autocomplaciente que poco ayuda al crecimiento y al desarrollo de un grupo social y de sus miembros.

En este marco, Restrepo (2004) se pregunta acerca del sentido, la legitimidad y la conveniencia del cuestionamiento, de parte de los investigadores, del esencialismo cultural de los grupos étnicos que estudian. Pone en duda que tal cuestionamiento signifique necesariamente un desempoderamiento de los movimientos sociales que expresan los intereses de esos grupos. Al mismo tiempo, critica la noción de que la defensa de las narrativas esencialistas favorezca a los grupos subordinados. El conocimiento experto no se libera de su carga hegemónica al ponerse a disposición de los discursos de los grupos subalternos; todo lo contrario, puede contribuir a reforzar las posiciones de subordinación de donde provienen esos discursos. Restrepo cree que una visión constructivista e histórica de la identidad y de la cultura pueda contribuir a favorecer "nuevas políticas de las etnicidades (en plural), desvinculadas de un sujeto étnico predeterminado y trascendental" (Restrepo 2004:244), es decir, que no tengan obligatoriamente que pasar por la esencialización de la identidad para lograr su legitimación política.

Recogiendo los aportes de tales discusiones, se quieren señalar aquí algunos aspectos problemáticos de un proyecto educativo basado en una visión esencialista de la cultura mapuche. El objetivo de este análisis no es, en ningún modo, cuestionar el valor y la legitimidad del proyecto educativo per se sino mostrar que su componente esencialista conlleva más riesgos que beneficios para el logro del mismo.

En primer lugar, hay que señalar que, pese al carácter inclusivo de la escuela, en términos de su no discriminación por pertenencia étnica o religiosa, su modelo formativo es excluyente al estar dirigido a un individuo mapuche que haga de "lo propio", lo legítimamente reconocido como mapuche desde una visión esencialista, como única fuente de sentido para su vida. A través de la formación se transmite un ideal de mapuche en el cual los niños evangélicos presentes en la escuela, por ejemplo, no pueden identificarse, dado que, en principio, estarían estructuralmente imposibilitados de alcanzar los objetivos educacionales que se propone la escuela, por su condición de practicantes de una religión ajena a la cultura mapuche. De hecho, así lo están entendiendo los niños evangélicos que en las entrevistas declararon no ser mapuches debido a su pertenencia religiosa. Por otra parte, ese modelo de mapuche es inaccesible, en teoría o en el discurso esencialista, también para todos los niños mapuches que viven su condición de "seres interculturales". Como la práctica intercultural es, tal como admiten los mismos profesores, un hecho inevitable, la paradójica consecuencia de formar en pos de ese modelo es la generación de una situación dilemática para esos niños, divididos entre el llamado a su esencia mapuche y su doble pertenencia. Esa división interna puede derivar en dos posibles caminos en el momento crucial de salida de la escuela para ingresar a la enseñanza media: uno es el del distanciamiento de un proyecto personal y colectivo de fortalecimiento de la identidad mapuche. La formación en la "conciencia de ser mapuche" puede no ser suficiente para contrarrestar la inmersión en la cultura nacional. La idea, heredada de una formación esencialista, de incompatibilidad de un proyecto de vida mapuche con el mundo no mapuche fácilmente podría desembocar en la opción por abandonar ese proyecto de vida y, con él, cualquier intento de vivir la propia identidad cultural y defender los derechos colectivos.

Es necesario recordar que el esencialismo cultural se enseña en la escuela We Newen solo a nivel discursivo porque la vida y práctica cotidiana de la escuela es, como se ha mencionado, significativamente intercultural. Desde una visión de aprendizaje como práctica y participación en 
actividades relacionales, la interculturalidad vivida en la cotidianidad de la vida escolar puede jugar un papel determinante a la hora de tener que optar entre perseguir el proyecto purista de vida mapuche o el camino de la "contaminación cultural".

El otro camino posible que se perfilaría desde una formación cultural de corte esencialista es el de una existencia muy atormentada para los niños mapuches quienes, aun manteniendo su opción por el proyecto de vida mapuche, vivirían en el drama de la imposibilidad de llevarlo a cabo debido a la necesidad de vivir entre dos mundos. En otras palabras, lo que puede traer complicaciones para los niños, desde el punto de vista psicológico, parece ser no el hecho de desplazarse entre dos mundos culturales y pertenecer a ambos, sino la misma problematización que se hace de ello.

Como sugiere Restrepo (2004) no es necesario el fundamentalismo étnico o el planteamiento de una esencia cultural para defender los derechos de autodeterminación como pueblo. En la misma línea, propongo que no es inevitable transmitir una idea esencialista del ser mapuche para llevar adelante un proyecto educativo orientado al fortalecimiento de la identidad étnica y el empoderamiento social y político. La escuela We Newen desarrolla un trabajo ejemplar al combinar la vivencia y la práctica de la cultura y lengua mapuche, aun con los límites impuestos por el formato escolar, con la formación de una "conciencia étnica". Es la demostración que la vía de la gestión indígena en el campo de la educación, tal como plantea el Convenio 169 de la Organización Internacional del Trabajo (OIT), merece ser perseguida como una modalidad alternativa o complementaria a la EIB. En este marco se insinúa que disolver el discurso esencialista para dejar espacio a la práctica mapuche e intercultural en el ámbito escolar pueda ser beneficioso para un proyecto educativo que apunte al fortalecimiento de la identidad étnica en el mundo contemporáneo.

Agradecimientos: Mis agradecimientos van, en primer lugar, al Programa Fondecyt por haberme otorgado financiamiento para realizar esta investigación. En segundo lugar, agradezco infinitamente a los kimeltuchefe de la escuela We Newen por su acogida y por haberme concedido conocer de cerca su valioso proyecto. También gracias a Consuelo Telechea y Natalia Caniguan por las ricas conversaciones de las cuales se ha nutrido este trabajo y a los evaluadores anónimos de Chungara Revista de Antropología Chilena, por sus valiosos comentarios y sugerencias.

\section{Referencias Citadas}

Álvarez-Santullano, P., A. Alves, A. Forno, R. Rivera y P. Fuenzalida 2011. Saberes y anclajes de la escuela intercultural en contexto mapuche: silencios, intermitencias y estrategias en la transmisión del legado histórico. Alpha 32:127-148.

Álvarez-Santullano, P., A. Forno y R. Rivera 2007. La educación intercultural en el discurso directivo docente mapuche: posicionamiento desde los márgenes. En Patrimonio Cultural Mapunche, editado por T. Duran, D. Catriquir, A. Hernández, pp. 283-295. Editorial UC Temuco, Temuco.

Ansión, J. 2007. La interculturalidad y los desafíos de una nueva forma de ciudadanía. En Educar en Ciudadanía Intercultural, editado por J. Ansion y F. Tubino, pp. 37-62. Fondo Editorial Pontifica Universidad Católica del Perú, Lima.

Benedict, R. 1934. Patterns of Culture. Houghton Mifflin, Boston.

Canclini, N. 2006. El consumo cultural: una propuesta teórica. En El Consumo Cultural en América Latina. Construcción Teórica y Líneas de Investigación, editado por G. Sunkel, pp. 72-95. Convenio Andrés Bello, Bogotá.

Dietz, G. 2012. Multiculturalismo, Interculturalidad y Diversidad en Educación. Una Aproximación Antropológica. Fondo de Cultura Económica, México D.F.
Durán, T. 2007. Interculturalidad en Educación: Discontinuidades y reiteraciones de un ayer y hoy en territorio mapuche. En Patrimonio Cultural Mapunche, Vol. II, editado por T. Durán, D. Catriquir, A. Hernández, pp. 175-193. Editorial UC Temuco, Temuco.

Forno, A., P. Álvarez-Santullano y R. Rivera 2009. Entre el edificio y el currículum de la interculturalidad: una mirada antropológica a la educación actual en territorio mapuche-huilliche. Chungara Revista de Antropología Chilena 41:287-298.

Fuenzalida, P. y M. Casas 2010. La Educación Intercultural Bilingüe como campo de tensión política entre el Mundo Mapuche-Williche y el Estado. Revista Lider 16:7384.

Grillo, R. 2003. Cultural essentialism and cultural anxiety. Anthropological Theory 3:157-173.

Hviding, E. 1993. Indigenous essentialism? Simplifying customary land ownership in New Georgia, Solomon Islands. Bijdragen tot de Taal-, Land-en Volkenkunde 149:802-824.

Lave, J. y E. Wenger 1991. Situated Learning: Legitimate Peripheral Participation. Cambridge University Press, New York.

López, L. 2011. Top-down and bottom-up: Counterpoised visions of bilingual intercultural education in Latin America. En Can 
Schools Save Indigenous Languages?, editado por N. Hornberger, pp. 42-65. Palgrave Macmillan, New York.

Luna, L. 2007. Un Mundo entre Dos Mundos. Las Relaciones entre el Pueblo Mapuche y el Estado Chileno desde la Perspectiva del Desarrollo y de los Cambios Socioculturales. Ediciones Universidad Católica de Chile, Santiago.

Mattío, E. 2009. ¿Esencialismo estratégico? Un examen crítico de sus limitaciones políticas. Construyendo Nuestra Interculturalidad 4:1-11.

Mead, M. 1928. Coming of Age in Samoa: A Psychological Study of Primitive Youth for Western Civilization. Blue Ribbon Books, New York.

Peña-Cortés, F., O. Almendra, M. Rojas-Maturana 2010. Enfoque geográfico territorial de la educación intercultural. En Interculturalidad en Contexto Mapuche, editado por D. Quilaqueo, C. Aníbal y S. Quintriqueo, pp. 241-256. Editorial de la Universidad Nacional del Comahue, Neuquén.

Quidel, G. 2011. Estrategias de enseñanza de la lengua mapunzugun en el marco del PEIB Mineduc-Origenes (Comuna de Padre las Casas, IX Región-Chile). Cuadernos Interculturales 16:61-80.

Quilaqueo, D. y S. Quintriqueo 2010. Saberes educativos mapuches: un análisis desde la perpectiva de los kimches. Polis 26:2-17.

Quilaqueo, D., S. Quintriqueo, H. Torres y G. Muñoz 2014. Saberes educativos mapuches: aportes epistémicos para un enfoque de educación intercultural. Chungara Revista de Antropología Chilena 46:271-283.

Quintriqueo, S. y H. Torres 2012. Distancia entre el conocimiento mapuche y el conocimiento escolar en contexto mapuche. Revista Electrónica de Investigación Educativa 14:16-33.
Restrepo, E. 2004. Esencialismo étnico y movilización política: tensiones en las relaciones entre saber y poder. En Gente Negra en Colombia. Dinámicas Sociopolíticas en Cali y el Pacífico, editado por O. Barbary y F. Urrea pp. 227-244. Editorial Lealon, Medellín.

Rockwell, E. 2011. Le realtà latinoamericane e gli studi etnografici sui processi educativi. En Antropologia ed Educazione in America Latina, editado por F. Gobbo y C. Tallè, pp. 27-41. Centro d'Informazione e Stampa Universitaria, Roma.

Rockwell, E. y A. Gomes 2009. Introduction to the Special Issue: rethinking indigenous education from a Latin American Perspective. Anthropology \& Education Quarterly 40:97-109.

Rother, T. 2005. Conflicto intercultural y educación en Chile: Desafíos y problemas de la Educación Intercultural Bilingüe (EIB) para el Pueblo Mapuche. Revista Austral de Ciencias Sociales 9:71-84.

Spindler, G. (ed.) 1955. Education and Anthropology. Stanford University Press, California.

Spivak, G. 1987. In Other Worlds. Essays in Cultural Politics. Methuen, New York.

Tallè, C. 2011. La scuola, gli indigeni e lo Stato in America Latina, ovvero Bourdieu e la riproduzione inceppata. En Antropologia ed Educazione in America Latina, editado por F. Gobbo y C. Tallè, pp. 43-68. Centro d'Informazione e Stampa Universitaria, Roma.

Tosta, S. 2013. Cruzando fronteiras - entre a Antropologia e a Educação no Brasil e na Argentina. Pro-Posições 24:95-107.

Verkuyten, M. 2003. Discourses about ethnic group (de-) essentialism: Oppressive and progressive aspects. British Journal of Social Psychology 42:371-391.

Wenger, E. 1998. Communities of Practice: Learning, Meaning, and Identity. Cambridge University Press, New York.

\section{Notas}

1 La investigación en la cual está basado este artículo es el proyecto Fondecyt de Iniciación N.11110390 “Aprender en diferentes comunidades de práctica: estudio etnográfico comparativo de la práctica social de una "escuela indígena" rural y una "escuela no indígena" urbana en la región de la Araucanía", 2011-2014.

2 En este artículo se habla de "niño" y "niños" en sentido amplio que incluye tanto el género femenino como el masculino.

3 Por razones de confidencialidad, en este trabajo se refiere a la escuela, la comunidad y a los profesores por medio de pseudónimos. También, por el compromiso de anonimato asumido con la escuela no se entrega información más específica sobre la ubicación del establecimiento y se omiten antecedentes contextuales (geográficos, demográficos, etc.) que harían fácilmente identificable la escuela en cuestión.

4 Contrasta esta postura con la de algunos "mapuches urbanos" quienes ven en la interculturalidad o pertenencia a dos mundos una vía ineludible para el reposicionamiento de la identidad étnica. Véase Luna (2007:126). 
